\title{
Elevated serum levels of galectin-3 binding protein is associated with insulin resistance in non-diabeteic women after menopause
}

\section{Yun Chen}

Affiliated Hangzhou People's First Hospital of Zhejiang University School of Medicine

\section{Sayantana Das}

North Middlesex University Hospital NHS Trust

\section{Guangchao Zhuo}

Affiliated Hangzhou People's First Hospital of Zhejiang University School of Medicine

Hong Cai ( $\nabla$ jikang_ch@126.com )

Affiliated Hangzhou People's First Hospital of Zhejiang University School of Medicine https://orcid.org/0000-0001-7789-7133

\section{Research article}

Keywords: Menopause, Insulin resistance, Galectin-3 binding protein

Posted Date: March 9th, 2020

DOI: https://doi.org/10.21203/rs.3.rs-16399/v1

License: (9) This work is licensed under a Creative Commons Attribution 4.0 International License. Read Full License 


\section{Abstract}

Background Galectin-3 binding protein (GAL-3BP) is one of the major fucosylated glycoprotein family members, and is implicated in nonalcoholic fatty liver disease, lipid dysfunctions and coronary artery disease recently. Here we analyzed the serum concentrations of GAL-3BP in postmenopausal women, to evaluate the association of circulating GAL-3BP and insulin resistance in female after menopause.

Method We evaluated serum levels of Gal-3BP in sixty-two non-diabetic women in menopausal status for at least one year. The clinical features, biochemistry profiles and homeostasis model assessment of insulin resistance (HOMA-IR) were obtained routinely.

Results Gal-3BP levels increased in women with higher HOMA-IR values and was positively correlated to HOMA-IR. It was also an independent risk factors for HOMA-IR and showed the most influence on HOMA-IR comparing to FPG, TG, age and BMI. The cutoff value of serum Gal-3BP level was $2234.3150 \mathrm{ng} / \mathrm{ml}$ with the area under the ROC curve (AUC) 0.68 (HOMA-IR 1.5), 0.81 (HOMAIR 2.0) and 0.93 (HOMA-IR 2.5).

Conclusions Serum levels of Gal-3BP is associated with impaired insulin sensitivity in non-diabetic menopausal women.

\section{Introduction}

Insulin resistance is a complicated condition in which the insulin sensitivity of primary metabolic tissues, including the liver, white adipose tissue and skeletal muscle, is impaired, and insulin resistance is considered to be one of the major causes of type 2 diabetes and cardiovascular diseases. Menopausal women who have ceased menstruating for at least one year have a markedly higher probability of developing insulin resistance than age-matched premenopausal women, with the prevalence most commonly estimated at the level of $31 \%-55 \%[1,2]$. There is growing evidence indicating that hormone fluctuation triggered by menopause, especially decreasing levels of oestrogen in the circulation, may lead to increased visceral adipose deposition and systemic inflammatory responses, but the underlying mechanism remains unclear in many aspects.

Galectin-3 binding protein (Gal-3BP), one of the major fucosylated glycoprotein family members, appears to be implicated in the immune response and in cytotoxicity $[3,4]$. Recently, several clinical investigations have suggested that Gal-3BP is related to metabolic dysfunctions ranging from non-alcoholic fatty liver disease to atherosclerosis [5-7]. However, few studies have focused on the relationship between insulin resistance and Gal-3BP, and the results remain controversial $[8,9]$. For this reason, we conducted this study focused on the associations between Gal-3BP and insulin homeostasis and the relevance of Gal-3BP as a biomarker of insulin resistance in the menopausal population.

\section{Materials And Methods}

\subsection{Subjects}

Sixty-two women after menopause were recruited from the Physical Examination Centre of the Affiliated Hangzhou First People's Hospital, Zhejiang University School of Medicine, between January and April 2019. The study was approved by institutional review boards at the Affiliated Hangzhou First People's Hospital, Zhejiang University School of Medicine.

Exclusion criteria were pre- and peri-menopausal status; acute infection phase; a history of autoimmune, hepatic, renal, malignant tumoral or psychiatric diseases; a history of diabetes or heart stents; and the intake of a female hormone that is known to affect circulating sex steroids within the three months prior to the study.

\subsection{Anthropometric evaluation}

Participants were invited to the Physical Examination Centre between 8:00 and 10:00 am after overnight fasting. Anthropometric measurements, such as age, weight and height, were collected by trained personnel. BMI was calculated as weight in kilograms divided by height in metres squared. Blood pressure was measured in a resting state. 
Venous blood samples were collected and kept at room temperature for $30 \mathrm{~min}$ and then centrifuged at $1500 \times \mathrm{g}$ and $4{ }^{\circ} \mathrm{C}$ for 15 min within $5 \mathrm{~h}$ of collection. Biochemical variables such as alanine transaminase (ALT), triglycerides (TG), total cholesterol (TC), high-density lipoprotein cholesterol (HDL-C), low-density lipoprotein cholesterol (LDL-C), fasting plasma glucose (FPG), and fasting insulin (FINS) were determined according to standard laboratory methods.

Homeostatic model assessment of insulin resistance (HOMA-IR) indices were calculated as follows: HOMA-IR index = fasting glucose $(\mathrm{mmol} / \mathrm{dl}) \times$ fasting insulin $(\mu \mathrm{mol} / \mathrm{L}) / 22.5$.

\subsection{Measurement of circulating Gal3-BP by ELISA}

Serum Gal-3BP levels were measured with an enzyme immunoassay kit according to standard instructions (Human LAGLS3BP ELISA Kit, RayBio Inc.).

All assays were performed in duplicate, and average data were collected.

\subsection{Statistical analysis}

Statistical Package for the Social Sciences software (SPSS Inc. version 22.0, Armonk, USA) and GraphPad Prism (version 6.0, CA, USA) were used to analyse the data. The Kolmogorov-Smirnov test was used for normality evaluation. Normally distributed data are expressed as the mean $\pm S D$. Data not normally distributed were expressed as the median $\left(25^{\text {th }}-75^{\text {th }}\right.$ IQR). Before performing further analyses, non-normally distributed parameters were log-, square-, or normal-score- transformed. One-way ANOVA was performed for multi-group comparisons. The Kruskal-Wallis test was used for non-normally distributed data. Pearson's correlation analysis was used to determine the correlation of serum Gal-3BP with demographic and biochemical parameters. Multivariate analysis was performed by logistic regression to observe variables influencing the risk for insulin resistance adjusted for age and $\mathrm{BMI}$. The diagnostic performance of Gal-3BP was analysed by receiver operating characteristic (ROC) curves and the area under the ROC curve (AUC). The Youden index was used to identify the optimal cut-off points. Probability values were 2 tailed, and a $p$ value $<0.05$ was considered significant.

\section{Results}

\subsection{Clinical, metabolic and hormonal features of the study population.}

The parameters of clinical and biochemistry were given in four subgroups according to the quartiles of the HOMA-IR index, as shown in Table 1 and Fig. 1. Age, BMI, SBP, DBP, and circulating levels of FPG, FINS and ALT were significantly different among the four subgroups, while SBP and serum levels of TC, TG, HDL-C, and LDL-C were similar. Moreover, serum levels of Gal-3BP in the fourth quartile of HOMA-IR index subgroups were significantly higher than those of lower HOMA-IR index quartiles $(p<0.05)$.

\subsection{Association between Gal-3BP concentrations and metabolic parameters.}

We performed Pearson's correlation test in all subjects. The results showed that serum Gal-3BP levels were positively associated with fasting insulin $(r=0.517, p=0.000)$ and the HOMA-IR index $(r=0.513, p=0.000)$. There was a significant linear increase in Gal3BP expression with increasing quartiles of the HOMA-IR index. The data are summarized in Table 2 and Fig. 2.

\subsection{The influence of serum Gal-3BP levels on the HOMA-IR index in women after menopause.}

Multiple linear regression analysis showed that Gal-3BP, FPG, TG, age and BMI were independent risk factors for a high HOMA-IR index. Serum levels of Gal-3BP showed the most influence on the HOMA-IR index. The data are listed in Table 3.

\subsection{Ability of serum Gal-3BP levels to distinguish insulin-resistant individuals from all subjects.}

We investigated the ability of serum Gal-3BP levels to distinguish insulin-resistant patients from all individuals using the ROC curve shown in Table 4 and Fig. 3. Since there was great variability in the optimal reference range of the HOMA-IR index, we 
obtained three cut-off values, including $1.5,2.0$, and 2.5 , as suggested by several studies [10-15]. The cut-off value of Gal-3BP was $2234.3150 \mathrm{ng} / \mathrm{ml}$.

\section{Discussion}

The present work focused on the expression of Gal-3BP in non-diabetic menopausal women. Our results consistently indicated that a higher level of Gal-3BP was associated with increased insulin resistance. This finding was in line with data from previous in vivo and in vitro studies that showed increased expression of Gal-3BP in different phenotypes of metabolic syndrome, including non-alcoholic fatty liver disease, obesity and silent atherosclerosis [16,17]. However, in contrast to some previous studies, our data failed to show a significant correlation between Gal-3BP and clinical lipid profiles or age in women after menopause $[9,18]$. This may be due to differences in study subjects owing to the selective inclusive criteria and may imply that Gal-3BP is more associated with adipose dysfunction than with fat deposition [19].

Multiple linear regression analysis showed that Gal-3BP was an independent and significant determinant for impaired insulin sensitivity. Furthermore, Gal-3BP non-invasively and reliably predicted insulin sensitivity on the basis of the several tested HOMAIR index cut-off values in our study. Considering that circulating Gal-3BP increased when insulin dynamics were impaired much earlier than clinical diabetes diagnosis was confirmed, it is plausible that Gal-3BP is associated with the onset of insulin resistance and has an impact on the pathogenesis of type 2 diabetes mellitus.

As a binding protein, Gal-3BP binds specifically to human macrophage-associated lectins and is implicated in the immune response associated with natural killer (NK) and lymphokine-activated killer (LAK) cell cytotoxicity. Gal-3BP is suggested to be a major upstream molecule in the activation of inflammatory factors, including IL-6 and TNF-a, by playing an important role in macrophage polarization and activation. In addition, several investigations have suggested a strong link between Gal-3BP levels and metabolic disorders, consistent with systemic pro-inflammation, by influencing inflammatory signalling pathways, including JNK-STAT and IKKB/NF-KB [20-23]. There is solid evidence that insulin resistance is related to inflammation with both the fluctuation of cytokines and the relevant signalling pathways. For example, IL-6 is reported to induce insulin resistance by impairing the synthesis of glycogen and enhancing TLR-4 gene expression through the activation of STAT3 [24]. Menopausetriggered insulin resistance is also considered to partly rely on the decreased protective effect of oestrogen on the inflammatory response. Interestingly, Eva Olga Melin et al found that high Gal-3BP levels were associated with HbA1c only in women. These findings suggest that Gal-3BP may be associated with sex hormone fluctuation and have an impact on insulin resistance by systemic immune regulation. Further studies on the relationship between sex hormones and Gal-3BP expression are needed.

Some limitations of this work must be taken into consideration. First, the design of the observational study cannot implicate the cause-and-effect relationship. Second, the diagnostic criteria for insulin resistance were mainly based on HOMA-IR indices and not hyperinsulinaemic-euglycaemic clamp analyses. Finally, due to the small number of subjects, the trials may have weaknesses that could result in bias.

Despite these limitations and to our knowledge, this study found a significant and close relationship between Gal-3BP and insulin resistance in menopausal women, but the underlying mechanisms need further investigation, and the relationship between Gal3BP and sex hormones is necessary and important to be clarified in future investigations.

\section{Declarations}

\section{Ethics approval and consent to participate}

The study protocol was approved by the Ethical Committee of Affiliated Hangzhou First People's Hospital, Zhejiang University School of Medicine. Written informed consent was obtained from all participants in the study. The study was conducted according to the Declaration of Helsinki.

\section{Consent for publication}

Page $4 / 11$ 
Not applicable.

\section{Competing interests}

The authors declare that there are no competing interests.

\section{Funding}

This study was supported by the Major Science and Technology Programme and Key Projects of the Science and Technology Department of Zhejiang Province, China (grant 2014C03044-1).

\section{Authors' contributions}

Cai has full access to the data in the study and takes responsibility for the integrity of the data and the accuracy of the data analyses.

1) conceived and designed the experiments: Cai.

2) performed the experiments: Chen, Zhuo and Cai.

3) analysed and interpreted the data: Chen and Das.

4) contributed reagents, materials, analytical tools or data: Chen and Cai.

5) wrote the paper: Chen and Das.

\section{Acknowledgements}

The authors thank Professor Yueming Chen for valuable discussion and the staff of the central laboratory for assistance with the experiments.

\section{Availability of data and materials}

We are currently planning a related study. Therefore, the data and material are not shared in their current state. However, the datasets used and/or analysed during the current study are available from the corresponding author on reasonable request.

\section{References}

1. Stachowiak G, Pertynski T, Pertynskamarczewska M, et al. Metabolic disorders in menopause[J]. Przeglad Menopauzalny, 2015, 14(1): 59-64.

2. Khanam M A, Qiu C, Lindeboom W, et al. The metabolic syndrome: prevalence, associated factors, and impact on survival among older persons in rural Bangladesh[J]. PLOS ONE, 2011, 6(6): 1-7.

3. Koths K E, Taylor E W, Halenbeck R, et al. Cloning and characterization of a human Mac-2-binding protein, a new member of the superfamily defined by the macrophage scavenger receptor cysteine-rich domain[J]. Journal of Biological Chemistry, 1993, 268(19): 14245-14249.

4. Nielsen $C T$, Ostergaard $O$, Rekvig $O P$, et al. Galectin-3 binding protein links circulating microparticles with electron dense glomerular deposits in lupus nephritis[J]. Lupus, 2015, 24(11): 1150-1160.

5. Gleissner C A, Erbel C, Linden F, et al. Galectin-3 binding protein, coronary artery disease and cardiovascular mortality: Insights from the LURIC study[J]. Atherosclerosis, 2017: 121-129.

6. Sugiura T, Dohi Y, Takase H, et al. Serum levels of Mac-2 binding protein increase with cardiovascular risk and reflect silent atherosclerosis[J]. Atherosclerosis, 2016: 192-196.

7. Tamaki N, Higuchi M, Kurosaki M, et al. Wisteria floribunda agglutinin-positive mac-2 binding protein as an age-independent fibrosis marker in nonalcoholic fatty liver disease[J]. Scientific Reports, 2019, 9(1):10109-10117. 
8. Choi R, Oh Y, Lee S, et al. Evaluation of the Serum Mac-2 Bingding Protein Glycosylation Isomer Test used for Diagnosis and Monitoring of Liver Fibrosis and the Correlation of Mac-2 Binding Protein Glycosylation Isomer with Hemoglobin A1c[J]. Clin Lab, 2019,65(1).

9. Melin E O, Dereke J, Hillman M, et al. Female sex, high soluble CD163, and low HDL-cholesterol were associated with high galectin-3 binding protein in type 1 diabetes[J]. Biology of Sex Differences, 2019, 10(1):51-60.

10. Lee $\mathrm{C} \mathrm{H}$, Shih A Z, Woo Y C, et al. Optimal Cut-Offs of Homeostasis Model Assessment of Insulin Resistance (HOMA-IR) to Identify Dysglycemia and Type 2 Diabetes Mellitus: A 15-Year Prospective Study in Chinese[J]. PLOS ONE, 2016, 11(9).

11. Gayosodiz P, Oterogonzalez A, Rodriguezalvarez M X, et al. Insulin resistance (HOMA-IR) cut-off values and the metabolic syndrome in a general adult population: effect of gender and age: EPIRCE cross-sectional study[J]. BMC Endocrine Disorders, 2013, 13(1): 47-47.

12. Sb C V. Analysis of the Degree of Insulin Resistance in Post Menopausal Women by Using Skin Temperature Measurements and Fasting Insulin and Fasting Glucose Levels: A Case Control Study[J]. Journal of clinical and diagnostic research : JCDR, 2012, 6(10): 1644-1647.

13. Motamed N, Miresmail S J, Rabiee B, et al. Optimal cutoff points for HOMA-IR and QUICKI in the diagnosis of metabolic syndrome and non-alcoholic fatty liver disease: A population based study[J]. Journal of Diabetes and Its Complications, 2016, 30(2): 269-274.

14. Kim B, Choi H Y, Kim W, et al. The cut-off values of surrogate measures for insulin resistance in the Korean population according to the Korean Genome and Epidemiology Study (KOGES)[J]. PLOS ONE, 2018, 13(11).

15. Wiklund P, Zhang X, Pekkala $S$, et al. Insulin resistance is associated with altered amino acid metabolism and adipose tissue dysfunction in normoglycemic women[J]. Scientific Reports, 2016, 6(1): 24540-24540.

16. Dhana K, Braun K V, Nano J, et al. An Epigenome-Wide Association Study of Obesity-Related Traits[J]. American Journal of Epidemiology, 2018, 187(8): 1662-1669.

17. Sugiura T, Dohi Y, Takase H, et al. Serum levels of Mac-2 binding protein increase with cardiovascular risk and reflect silent atherosclerosis[J]. Atherosclerosis, 2016: 192-196.

18. Padilla J, Jenkins N T, Thorne P K, et al. Identification of genes whose expression is altered by obesity throughout the arterial tree[J]. Physiological Genomics, 2014, 46(22): 821-832.

19. Kapur S K, Katz A J. Review of the adipose derived stem cell secretome[J]. Biochimie, 2013, 95(12): 2222-2228.

20. Xu G, Xia Z, Deng F, et al. Inducible LGALS3BP/90K activates antiviral innate immune responses by targeting TRAF6 and TRAF3 complex[J]. PLOS Pathogens, 2019, 15(8).

21. Verhelst X, Dias A M, Colombel J, et al. Protein Glycosylation as a Diagnostic and Prognostic Marker of Chronic Inflammatory Gastrointestinal and Liver Diseases[J]. Gastroenterology, 2019, 158(1): 95-110.

22. Deroo E P, Wrobleski S K, Shea E, et al. The role of galectin-3 and galectin-3-binding protein in venous thrombosis[J]. Blood, 2015, 125(11): 1813-1821.

23. Fukaya $Y$, Shimada $H$, Wang L, et al. Identification of Galectin-3-binding Protein as a Factor Secreted by Tumor Cells That Stimulates Interleukin-6 Expression in the Bone Marrow Stroma[J]. Journal of Biological Chemistry, 2008, 283(27): 1857318581.

24. Chen L, Chen R, Wang H, et al. Mechanisms Linking Inflammation to Insulin Resistance[J]. International Journal of Endocrinology, 2015, (2015): 508409-508409.

\section{Tables}

Table.1 Demographic characteristics and physical and metabolic measurements overall and across quartiles of HOMA-IR indices 


\begin{tabular}{|c|c|c|c|c|c|c|}
\hline & \multirow{3}{*}{$\begin{array}{l}\text { Overall } \\
(n=62)\end{array}$} & \multicolumn{4}{|c|}{ Quartiles of HOMA-IR indices } & \multirow{3}{*}{$\begin{array}{l}p- \\
\text { value }\end{array}$} \\
\hline & & 4 & 3 & 2 & 1 & \\
\hline & & $(n=16)$ & $(n=16)$ & $(n=14)$ & $(n=16)$ & \\
\hline Age (years) & $58.2 \pm 5.5$ & $56.0 \pm 5.6$ & $61.3 \pm 4.7$ & $59.4 \pm 4.8$ & $56.3 \pm 5.5$ & 0.012 \\
\hline BMI $\left(\mathrm{kg} / \mathrm{m}^{2}\right)$ & $25.1 \pm 1.3$ & $25.8 \pm 1.1$ & $24.6 \pm 1.7$ & $25.2 \pm 0.9$ & $24.6 \pm 0.9$ & 0.016 \\
\hline SBP (mmHg) & $124.3 \pm 11.6$ & $131.0 \pm 5.1$ & $123.0 \pm 15.6$ & $121.0 \pm 11.0$ & $121.8 \pm 10.5$ & NS \\
\hline \multirow[t]{2}{*}{$\mathrm{DBP}(\mathrm{mmHg})$} & 73.0 & $79.0 \pm 5.3$ & $72.5 \pm 9.8$ & $67.1 \pm 7.0$ & $75.5 \pm 6.0$ & 0.000 \\
\hline & $(69.0-79.0)$ & & & & & \\
\hline \multirow[t]{2}{*}{ TC (mmol/L) } & 5.47 & $5.60 \pm 0.82$ & $5.53 \pm 0.71$ & $5.41 \pm 0.37$ & $5.70 \pm 1.11$ & NS \\
\hline & $(5.09-5.87)$ & & & & & \\
\hline \multirow[t]{2}{*}{ TG (mmol/L) } & 1.97 & $2.39 \pm 0.60$ & $1.92 \pm 0.74$ & $2.02 \pm 0.54$ & $1.87 \pm 0.50$ & NS \\
\hline & $(1.62-2.38)$ & & & & & \\
\hline \multirow{2}{*}{$\begin{array}{l}\text { HDL-C } \\
(\mathrm{mmol} / \mathrm{L})\end{array}$} & 1.20 & $1.29 \pm 0.27$ & $1.21 \pm 0.20$ & $1.26 \pm 0.20$ & $1.27 \pm 0.20$ & $N S$ \\
\hline & $(1.13-1.35)$ & & & & & \\
\hline \multirow{2}{*}{$\begin{array}{l}\text { LDL-C } \\
\text { (mmol/L) }\end{array}$} & 3.33 & $3.41 \pm 0.59$ & $3.43 \pm 0.54$ & $3.27 \pm 0.47$ & $3.43 \pm 0.86$ & NS \\
\hline & $(3.12-3.70)$ & & & & & \\
\hline \multirow[t]{2}{*}{ FPG (mmol/L) } & 4.95 & $5.12 \pm 0.46$ & $5.46 \pm 0.65$ & $4.98 \pm 0.30$ & $4.89 \pm 0.31$ & 0.005 \\
\hline & $(4.85-5.38)$ & & & & & \\
\hline \multirow[t]{2}{*}{ FINS (mU/L) } & 6.88 & $14.32 \pm 2.25$ & $8.46 \pm 1.51$ & $6.14 \pm 0.62$ & $4.19 \pm 0.50$ & 0.000 \\
\hline & $(4.78-11.34)$ & & & & & \\
\hline \multirow[t]{2}{*}{ HOMA-IR index } & 1.73 & $3.26 \pm 0.58$ & $2.02 \pm 0.23$ & $1.36 \pm 0.15$ & $0.91 \pm 0.12$ & 0.000 \\
\hline & $(1.05-2.42)$ & & & & & \\
\hline \multirow[t]{2}{*}{ ALT (U/L) } & 26.0 & $45.1 \pm 29.5$ & $27.5 \pm 12.4$ & $25.9 \pm 5.4$ & $28.1 \pm 12.4$ & 0.010 \\
\hline & $(22.0-38.0)$ & & & & & \\
\hline \multirow{2}{*}{$\begin{array}{l}\text { Gal-3BP } \\
\text { (ng/ml) }\end{array}$} & 1734.23 & $2916.72 \pm 1089.59$ & $1656.64 \pm 523.27$ & $1598.23 \pm 476.67$ & $1455.58 \pm 763.76$ & 0.000 \\
\hline & $\begin{array}{l}(1243.34- \\
2323.45)\end{array}$ & & & & & \\
\hline
\end{tabular}

Abbreviations: SBP systolic blood pressure; DBP diastolic blood pressure; TC total cholesterol; TG triglycerides; HDL-C high-density lipoprotein cholesterol; LDL-C low-density lipoprotein cholesterol; FPG fasting plasma glucose; FINS fasting insulin; HOMA-IR homeostasis model assessment-insulin resistance; ALT alanine transaminase; NS No significant difference.

Table 2. Correlation between Gal-3BP and anthropometric/metabolic parameters in all subjects

\begin{tabular}{|lllllllllllll|}
\hline variables & Age & BMI & SBP & DBP & TC & TG & $\begin{array}{l}\text { HDL- } \\
\text { C LDL-C }\end{array}$ & FPG & FINS & $\begin{array}{l}\text { HOMA- } \\
\text { IR }\end{array}$ & ALT \\
\hline $\mathbf{r}$ & -0.045 & 0.226 & 0.012 & -0.095 & -0.216 & -0.022 & 0.003 & -0.144 & -0.073 & 0.517 & 0.513 & -0.083 \\
\hline $\boldsymbol{p}$-value & 0.729 & 0.077 & 0.925 & 0.46 & 0.092 & 0.868 & 0.981 & 0.262 & 0.572 & $0.000 * *$ & $0.000 * *$ & 0.522 \\
\hline
\end{tabular}

** $p$-value $<0.01$. 
Table.3 The influence of factors on the HOMA-IR index by multiple regression analysis

\begin{tabular}{|lllllll|}
\hline Factor & \multicolumn{2}{l}{ Unstandardized Coefficients } & Standardized coefficients & t & \multirow{2}{*}{$p$-value } \\
\cline { 2 - 5 } & B & S.E. & Beta & & \\
\hline Age & 0.015 & 0.005 & 0.250 & 2.869 & $0.008^{\star *}$ \\
\hline BMI & 0.058 & 0.021 & 0.224 & 2.712 & $0.009^{\star *}$ \\
\hline SBP & 0.002 & 0.003 & 0.083 & 0.794 & 0.431 \\
\hline DBP & 0.086 & 0.035 & 0.247 & 2.484 & $0.016^{*}$ \\
\hline TC & -0.860 & 2.346 & -0.157 & -0.366 & 0.716 \\
\hline TG & 0.831 & 0.262 & 0.327 & 3.169 & $0.003^{\star *}$ \\
\hline HDL-C & 0.038 & 0.183 & 0.025 & 0.209 & 0.835 \\
\hline LDL-C & 0.075 & 0.208 & 0.138 & 0.358 & 0.722 \\
\hline FPG & 0.118 & 0.030 & 0.340 & 3.965 & $0.000^{\star *}$ \\
\hline Gal-3BP & 0.668 & 0.125 & 0.482 & 5.363 & $0.000^{\star *}$ \\
\hline ALT & 0.065 & 0.035 & 0.189 & 1.883 & 0.065 \\
\hline
\end{tabular}

** $p$-value $<0.01 ; * p$-value $<0.05$

Table 4. Quality of cut-off scores for the prediction of impaired insulin resistance

\begin{tabular}{|lllll|}
\hline HOMA-IR index cut-off & AUC $(\mathbf{9 5} \% \mathrm{Cl})$ & Gal-3BP cut-off & Sensitivity, \% & Specificity, \% \\
\hline $\mathbf{1 . 5}$ & $0.6752(0.5420-0.8085)$ & 2234.3150 & 42.1 & 83.3 \\
\hline $\mathbf{2 . 0}$ & $0.809(0.685-0.933)$ & 2234.3150 & 42.1 & 83.3 \\
\hline $\mathbf{2 . 5}$ & $0.927(0.862-0.992)$ & 2234.3150 & 100 & 84 \\
\hline
\end{tabular}

AUC area under the curve.

\section{Figures}




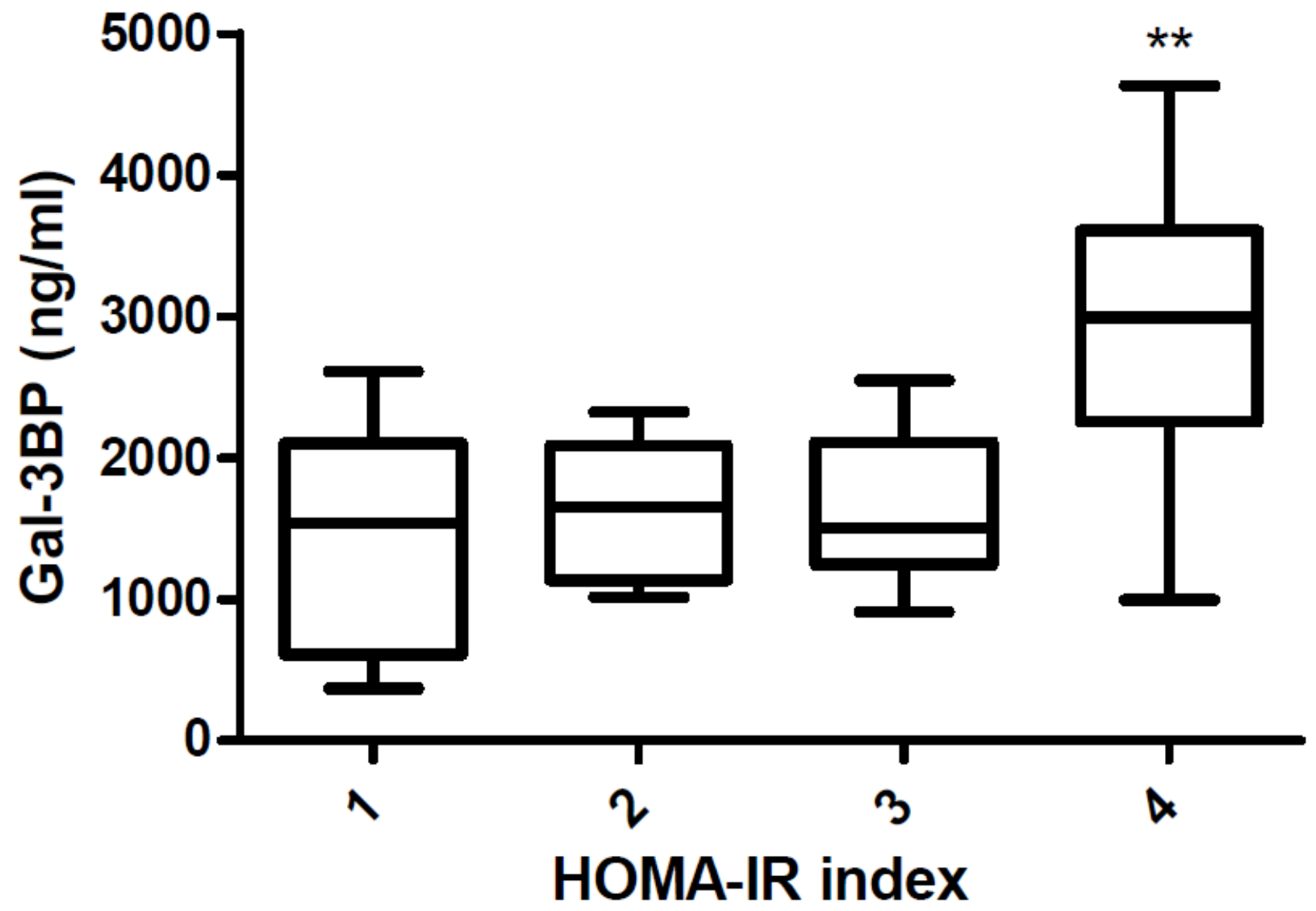

Figure 1

Serum levels of Gal-3BP in groups across quartiles of HOMA-IR indices. ** $p$ value $<0.01$. 


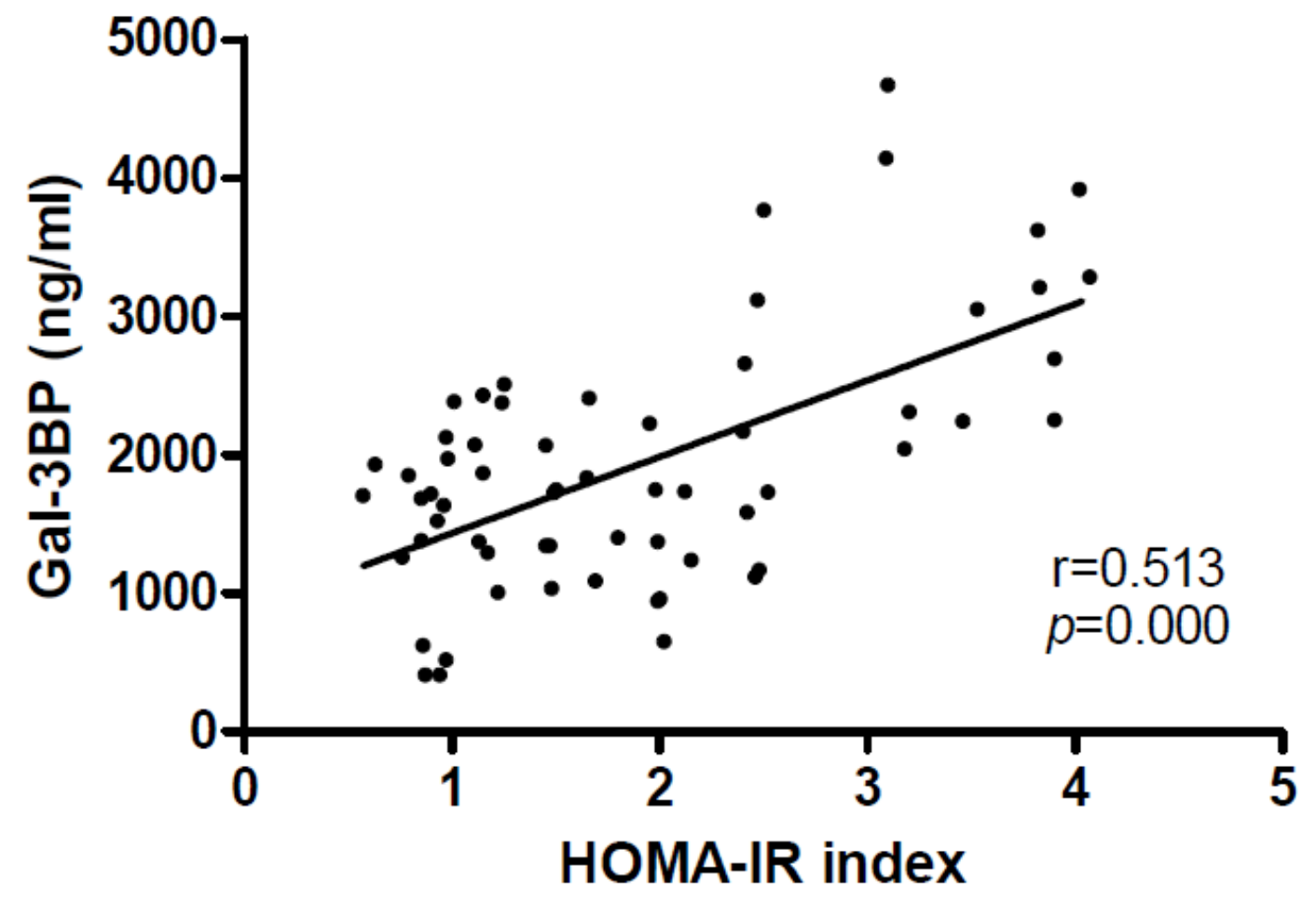

Figure 2

Positive correlation between serum Gal-3BP levels and HOMA-IR indices.

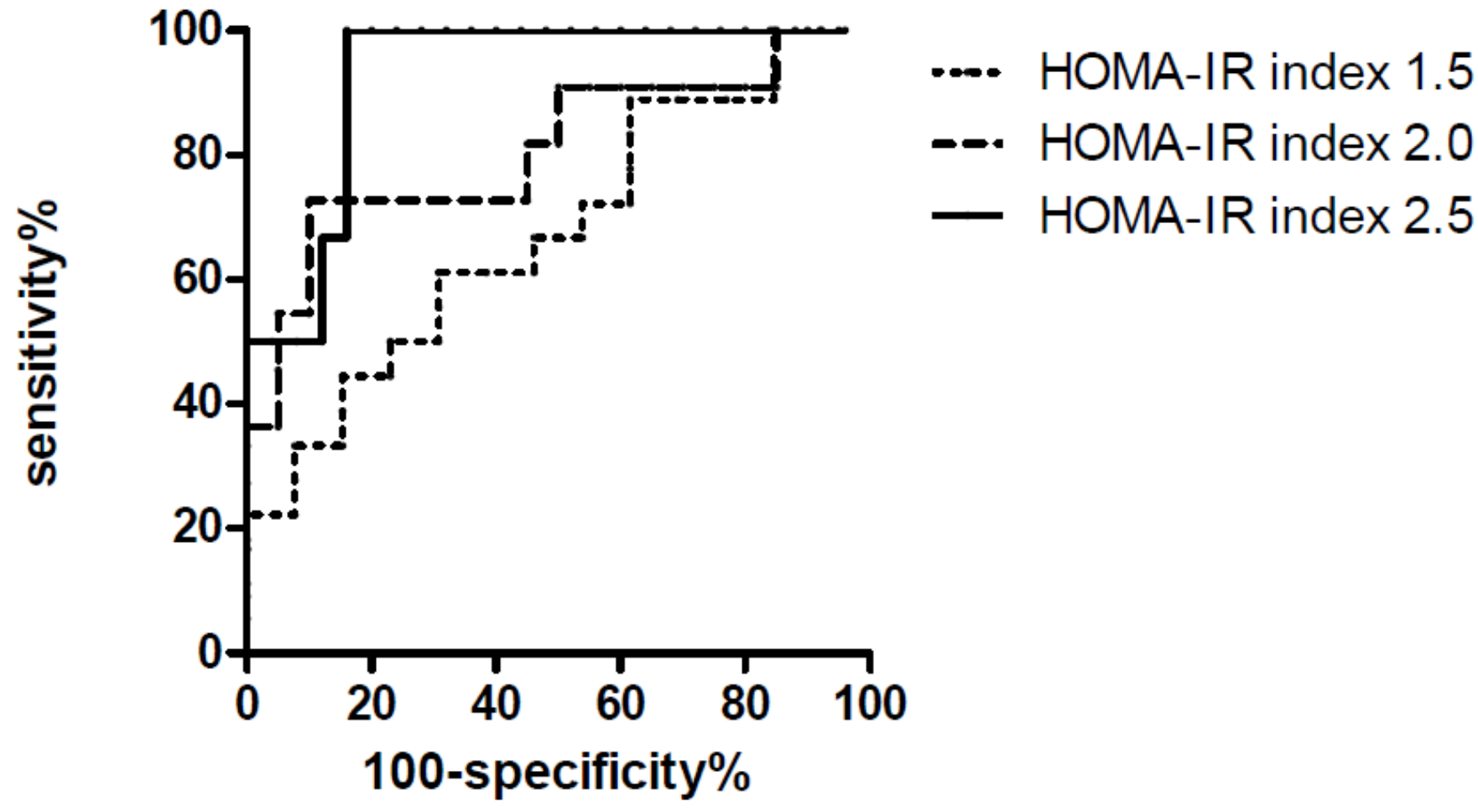


Figure 3

ROC curves for the discrimination of insulin-resistant patients from general subjects based on three cut-off values of the HOMA-IR index. 